\title{
LA REGULACIÓN JURÍDICA DE LOS SERVICIOS PÚBLICOS: \\ CASO DEL AGUA Y SANEAMIENTO EN EL PERÚ
}

\section{Resumen}

El presente artículo explica la regulación jurídica de los servicios públicos, señalando las funciones de quienes lo ejercen y mencionando la evolución de la función regulatoria del Estado tanto en la actividad pública como en el mercado. Por otro lado, analiza el fin de los órganos reguladores del Estado, haciendo particular énfasis en la regulación del servicio de agua y saneamiento en el Perú; es así que el autor explica el contexto histórico de los principales entes reguladores en este sector. Finalmente, el autor analiza las diferentes tendencias regulatorias del modelo peruano y cómo estas se encuentran fundamentadas en la regulación económica y social, así como en las actitudes del mercado.

\section{Summary}

The present article expia ins the legal regulation of public Services, slating the tasks of those who exercise il and mentioning the evolution of Slate regulalory function in the public activity and the global markel. Then, analvsis the final;ty of regulalory enlilies, wilh particular emphasis in the regulation of water Services and sanitation in Pertt: also the author explains the historie context of the principáis regulalory' enlilies in litis sector. Finally, lite author analyses the different regulalory trends of Peruvian ntodel and Itow these are grounded in economic and social regulation as well in global market altitudes.

\section{Sommaire}

Cet arlicle explique la réglementation juridique des Services publics, en notanI les foncüons de ceux qui I 'exercenl el menlionnant I 'évolution de la fonction régulalrice de I 'Elat, dans le public el dans le marché. D ature part, se tourne vers les organismes de réglementation de I 'Elal, avec un accent particulier sur la réglementation des Services d'eau el d'assainissemenl an Pérou, aussi / 'auleur explique le contexte historique des régulateurs clés dans ce secteur. Enfin. I 'auleur analvse les différentes tendances de modéle réglementaire dtt Pérou el de la facón donI ceux-ci son/ mis á la lene dans la régulation économique et sociale. ainsi que les aetions du marché. 


\section{LA REGULACIÓN DEL SERVICIO PÚBLICO}

\section{Generalidades:}

La regulación económica de los servicios públicos es una manifestación directa del poder de policía estatal, toda vez que alude a la capacidad del Estado, en empleo de su Ius Imperium, de imponer normas $u$ actos que han de ser acatados de forma ineludible; concretamente reconocida doctrina apunta que la misma;

"(...) condiciona, corrige o altera los parámetros naturales y espontáneos de los agentes económicos (...) incide sobre el acceso y salida de la actividad y afecta a las condiciones económicas en que la actividad se desarrolla: al quantum de producción, a las zonas o mercados que sirve cada empresa, a los precios o retribuciones que se perciben por ella, en suma, al negocio mismo en que la actividad consiste $(\ldots)^{\prime \prime \prime}$.

Consecuentemente, la finalidad esencial de la regulación consiste en la garantía de que la actividad prestadora del servicio público se mantenga en un estado constante de operatividad y que la ejecución de la misma logre una armonía entre la eficiencia económica y los parámetros legales establecidos para su desenvolvimiento. En este sentido, las funciones del regulador consisten en: (i) velar por la estabilidad de la prestación y verificar que la misma sea acorde con los estándares de calidad necesarios para la satisfacción de los usuarios; y, (ii) mantener el adecuado funcionamiento del mercado, materializando los fines del Estado Social y Democrático de Derecho. regulación que:

Por ello, debemos señalar respecto a las potestades y funciones estatales referidas a la

\footnotetext{
"(...) el estado no es solo una organización que interviene como garantía del ordenamiento jurídico, sino [más bien] determina o participa en el establecimiento de las reglas de juego, configurando de esta manera la vocación finalista por el bien común. Por ende, el Estado actúa como regulador y propiciador de los procesos económicos $(\ldots)^{\prime \prime 2}$.
}

Es importante notar en este extremo el tránsito que el Estado realizó para dejar de ser un ente que desarrollara, de modo concentrado, una serie de actividades económicas pasando a ser un Estado que actuara como regulador y es que la facultad que el Estado posee para insertar reglas en el mercado, propiciando el desarrollo y preservando aspectos de interés público alcanza un grado de fuerza vinculante similar a las potestades de participación económica ejercidas en antaño.

1 ARIÑo ORTIZ. Gaspar. Principios de Derecho Público Económico. Lima: ARA Editores E.I.R.L.. 2004. p. 336

2 Sentencia del Tribunal Constitucional recaída en el Exp. 7339-2006-PA/TC. Fundamento 18. 
Al respecto, la Corte Constitucional de Colombia nos brinda luces respecto a la función regulatoria del Estado señalando que:

"(...) es ejercida por una autoridad específicamente creada y concebida para fijar y ajustar de manera continua las reglas de juego a las cuales debe sujetarse una actividad determinada dentro de un sector socio-económico. (...) la actividad determinada sujeta a regulación, reviste una especial trascendencia en cuanto compromete el desarrollo del mercado en un ámbito donde, en mayor o menor medida, está envuelto el goce efectivo de los derechos de las personas y donde se juzga necesario adoptar medidas de protección social y de corrección de las fallas del respectivo mercado. (...) la autoridad reguladora dispone de instrumentos de regulación peculiares para el cumplimiento de su misión específica los cuales pueden ser de la más diversa naturaleza según el problema que ésta deba abordar, puesto que tales instrumentos van desde la mera recepción y divulgación de información (medida de comunicación), pasando por la intervención en los precios (medida económica) hasta la adopción de normas y la imposición de sanciones a quienes las infrinjan (medidas jurídicas). (...) la función de regulación es usualmente confiada a órganos con un mayor grado de independencia que el que tienen las entidades administrativas preexistentes en el respectivo país sometidas a controles jerárquicos o de tutela (...) la función de regulación está fundada en la necesidad de encontrar y mantener un equilibrio entre intereses legítimos contrapuestos en contextos socio-económicos de gran dinamismo de tal forma que, a pesar de los cambios frecuentes y acelerados que se presenten, el Estado disponga de instrumentos para orientar sostenidamente las actividades socio-económicas reguladas hacia los fines de interés general señalados por el constituyente y el legislador $(\ldots)^{\prime \prime \prime}$.

En base a lo expresado, correspondería que el Regulador pudiera desarrollar un grado de autonomía e institucionalidad considerable que permita un ejercicio real y eficaz de sus funciones. Si bien en el Perú existe una vinculación en cuanto a la adscripción del regulador a una instancia política, como lo es la Presidencia del Consejo de Ministros, sí resulta importante que aspectos vinculados a materia presupuestaria y de conformación de directorios gocen del reconocimiento y autonomía necesaria, significando ello un primer paso en el proceso de consolidación de autonomía institucional.

2. $\quad$ El rol del Regulador de servicio público:

Es oportuno precisar que:

"(...) [debemos] comprender a la regulación económica como el proceso a través del cual se actúa como un árbitro imparcial, ponderando, juzgando, decidiendo y arbitrando los distintos intereses de los [actores involucrados]; no agotándose en la sola función de re- 
dactar reglamentos..." ${ }^{4}$. Desde otro punto, se ha referido que el rol del regulador, entonces, puede ser visualizado en forma liminar, como el análisis, ex post, de esos costos [operativos que influyen en la tarifa] por eso es que - 110 sólo dado el tratamiento regulatorio de la base de la tarifa sino también por esta evaluación de los costos- se tomaría evidente que este modo de regulación depara una gran carga regulatoria. Así, el regulador deberá recolectar información sobre los costos de la empresa, y usualmente tendrá competencia para revisar la razonabilidad de dichos costos o su carácter excesivo. Consecuentemente, y según lo establezca el marco regulatorio respectivo, el regulador tendrá competencia para ejercer su escrutinio sobre dichos costos, pudiendo llegar a desaprobarlos a los fines de la fijación de las tarifas posibilitando que, en principio, y según se ha interpretado, no causa agravio constitucional $(. .,)^{\prime \prime 5}$.

En el marco de esta idea entendemos que la tarea regulatoria resulta bastante complicada no solo por la existencia de problemas vinculados a la falta de una institucionalidad desarrollada y a la presencia de una realidad de información asimétrica sino por la necesidad de que el regulador actúe como un ente que sintonice y establezca un equilibrio entre los intereses que se enfrentan en el mercado, tal como sucede con los intereses del Estado, de los usuarios y de los inversionistas.

Por otro lado, debemos recordar que la regulación económica del mercado guarda estrecha relación con el diseño constitucional de nuestro Estado previsto en el artículo $43^{\circ}$ de la Constitución Política del Perú de 1993, el cual nos define como un Estado Social y Democrático de Derecho. Al respecto, el Tribunal Constitucional del Perú ha referido que el mismo:

"(...) no obvia los principios y derechos básicos del Estado de Derecho, tales como la libertad, la seguridad, la propiedad privada y la igualdad ante la Ley; antes bien, pretende conseguir su mayor efectividad, dotándolos de una base y un contenido material, a partir del supuesto de que individuo y sociedad no son categorías aisladas y contradictorias, sino dos términos en implicación recíproca. En otras palabras, los redimensiona. otorgándoles, a su vez, un contenido "social". Justamente, es bajo el marco del Estado Social y Democrático de Derecho donde se configuran los fines de nuestro régimen económico en tanto economía social de mercado. De esta manera, el carácter "social" del régimen determina que el Estado no pueda permanecer indiferente ante las actividades económicas de los particulares, lo que, por cierto, en modo alguno supone la posibilidad de interferir de manera arbitraria e injustificadamente en el ámbito de libertad reservado a los agentes económicos. Y es que en una economía social de mercado, tanto los particulares como el 
Estado asumen deberes específicos; en el primer caso, el deber de ejercitar las referidas libertades económicas con responsabilidad social, mientras que, en el segundo, el deber de ejercer un rol vigilante, garantista y corrector, ante las deficiencias y fallos del mercado, y la actuación de los particulares $(, . .)^{\prime \prime 6}$.

Debemos caer en la cuenta que la regulación, sobre todo en el marco de un servicio público, no debe encontrarse dirigida a: "(...) [Ser] una herramienta de promoción de políticas sectoriales $(, . .)^{\prime \prime 7}$ sino más bien obedecer a una postura imparcial y autónoma de tinte político que permita que los objetivos que el regulador se plantee en su labor no se entremezclen con la tarea o manejo político y administrativa que ejercen algunos órganos del Estado.

En efecto, los organismos reguladores deben ser lo suficientemente fuertes para balancear las demandas de los distintos grupos de interés públicos o privados, ubicándose al medio de ellos, lo que implica un mandato claro $^{8}$, en consecuencia, requiere usualmente de una agencia independiente y autónoma que coadyuve a un desempeño óptimo de la industria. Un mal diseño de los mecanismos de regulación, entre los cuales se encuentran los reguladores, puede tener un efecto indeseable en la eficiencia y competitividad de la industria ${ }^{9}$.

Un sistema regulatorio óptimo se logrará otorgando la autonomía necesaria a la agencia reguladora, para que actúe eficientemente. El organismo regulador podrá enfrentar la politización potencial, al dotársele de condiciones que consolidan su conformación, estabilidad y recursos: (i) mandato independiente, (ii) estabilidad del directorio -autoridad de nombramiento y ejercicios fijos-, (iii) presupuesto autónomo, y (iv) Procedimientos de apelación.

En el Perú, los Organismos Reguladores de servicios públicos han sido creados en teoría, con autonomía administrativa, funcional, técnica económica y financiera ${ }^{10}$, para responder a mercados dinámicos y en permanente desarrollo. Su autonomía se encuentra sustentada, ya que participan como entes equilibrantes entre los agentes del mercado (Gobierno, empresas, usuarios), garantizando la provisión de los servicios públicos en condiciones de calidad y precio adecuados, en beneficio de la población. En ese aspecto:

Autonomía administrativa y funcional, significa contar con mecanismos precisos para nombrar y destituir funcionarios superiores.

\footnotetext{
$6 \quad$ Sentencia del Tribunal Constitucional recaída en el Exp.01963-2(K)6-AA/TC. 
Autonomía técnica, que permite aplicar estrictamente la ley, atendiendo exclusivamente a consideraciones profesionales, sin ningún espacio para la injerencia política.

Autonomía económica y financiera permite al organismo proveerse de los recursos necesarios y coadyuvar las otras dimensiones de la independencia. Libera a los reguladores del control presupuestario directo del gobierno, permitiéndole además, una utilización más autónoma y ajustada a sus intereses, para la constitución de un órganosno fiscalizador con alta capacidad técnica.

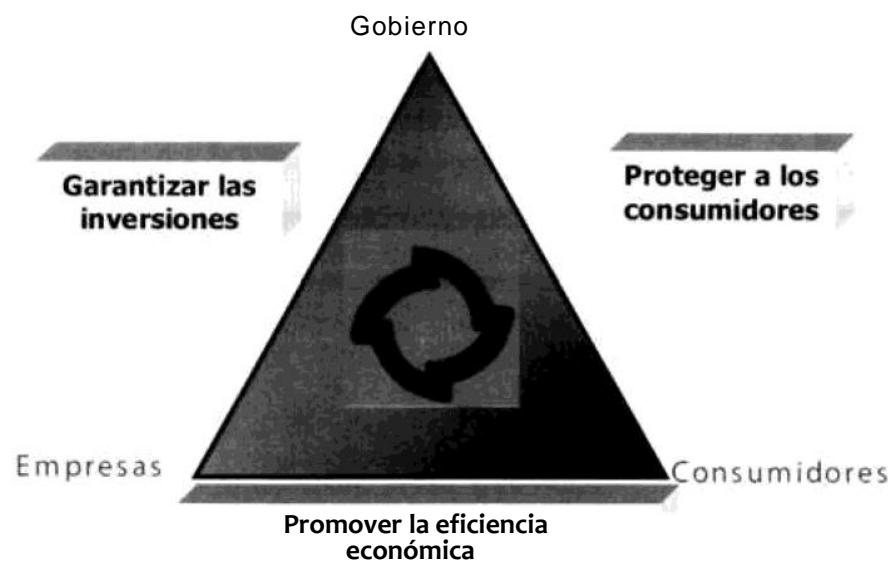

Fuentes: Smith (1997). Estacha y Martimort (1999). Ugaz (2003). Parker y Kirkpatnck (2002). Makaya (2001). Smith y Klein (1994). Gray (2001). IEA (2001) y Chisan et al (2001)

No obstante ello, en la práctica se ha adoptado en el país un concepto restringido de la regulación, el mismo que se identifica con la regulación desde la perspectiva económica, la cual entiende que la regulación es una interferencia del Estado en las actividades económicas calificadas como servicios públicos u obras de infraestructura. dirigida a controlar los monopolios naturales, bajo una forma de intervención o predeterminación de las reglas jurídicas aplicables a tales actividades".

La crítica de mayor gravedad a los organismos reguladores es la que pone de manifiesto su debilidad frente a la captura por los sectores regulados, la que puede

11 HUAPAYA TAPIA, Ramón. Algunos apuntes sobre las relaciones entre el Derecho Administrativo Económico y el concepto anglosajón de la regulación 
ocurrir tanto en monopolios o en mercados abiertos, ambos producto de la privatización. En efecto, en el Perú, los sectores de telecomunicaciones, transporte y energía han llegado a ser concesionados a diferencia del sector de agua y saneamiento el que aún se mantiene bajo el control estatal - municipal. Se viene advirtiendo con mayor frecuencia la constante presión del Gobierno de turno, la misma que incide sobre el ejercicio eficiente de funciones desde el regulador. Precisamente en este último escenario, los organismos reguladores comparten con varias entidades e instituciones su espacio regulatorio, el cual está sujeto a un compartimiento de poder e influencia desde el Congreso de la República, el Poder Judicial, el Poder Ejecutivo, entre otros agentes que permanentemente ejercen presión y usan su poder para tratar de influir en las decisiones del proceso regulatorio".

\section{3. $\quad$ La requlación del servicio de aqua v saneamiento en el Perú:}

En este extremo existe un documento" sobre la génesis del servicio público materia del presente estudio formulado por Lidia Oblitas de Ruiz para la Comisión Económica para América Latina (en adelante. CEPAL) en la que indica que a inicios de la década de 1980, los servicios de agua potable y saneamiento se condujeron de manera centralizada por el Poder Ejecutivo, siguiendo la tendencia a una planificación y a un manejo global de los servicios, modelo que era muy común entre la mayoría de países latinoamericanos, basado en el concepto de servicio público que debe ser financiado y subsidiado por el Estado, constituyendo el objetivo la cobertura total de los servicios por razones sanitarias, soslayando en absoluto el matiz empresarial.

El modelo centralizado, propio de la época, empieza a perder vigencia con el inicio del proceso de descentralización propugnado por la Constitución Política de 1979. En este contexto, el sector agua potable y saneamiento da un paso hacia una mayor descentralización con la creación de la empresa estatal Servicio Nacional de Abastecimiento de Agua Potable y Alcantarillado (en adelante, SENAPA) integrada por 15 empresas filiales, una de las cuales fue la empresa de Lima denominada Servicio de Agua Potable y Alcantarillado de Lima (en adelante, SEDAPAL) y 10 Unidades operativas distribuidas en todo el país, que fueron adquiriendo un manejo cada vez más independiente. Si bien con SENAPA se mantiene todavía el modelo centralizado en esta etapa se sustituye el concepto de obras, por el de servicio dirigido al usuario y se empieza a trabajar con un concepto más empresarial, aunque los servicios continúan dependiendo fuertemente de los recursos públicos.

Las responsabilidades rectoras, reguladoras y de prestación del servicio estuvieron concentradas en una sola institución SENAPA. En este período se crea también la Comisión de Tarifas de Agua Potable y Alcantarillado (en adelante, CORTAPA) responsable de revisar y aprobar los incrementos tarifarios en el ámbito urbano. Las tarifas se establecían desde un punto de vista contable con el objetivo de cubrir los gastos de administración, operación, mantenimiento y depreciación de instalaciones, y no se define una metodología para la regulación de tarifas.

A finales de esta etapa se dispone la transferencia de las Empresas Filiales y las Unidades Operativas, con excepción de la empresa de Lima, SEDAPAL, a los

\footnotetext{
12 Ibíd.

13 OBLITAS DE RUIZ, Lidia. "Servicios de Agua Potable y Saneamiento en el Perú: Beneficios potenciales y determinantes de éxito". Comisión Económica para América Latina. 2010
} 
Gobiernos Locales de forma desordenada y poco planificada sin la verificación de condiciones técnicas y económicas en los municipios para determinar si éstos se encontraban realmente preparados para asumir tal responsabilidad.

En la década de los noventa, el proceso histórico del sector agua potable y saneamiento, coincide con el ingreso de un nuevo gobierno que debió avocarse desde un inicio a revertir la grave crisis económica de fines del decenio anterior y a enfrentar la situación de lucha interna con el terrorismo. En este contexto, las primeras acciones se orientan a enfrentar el proceso acelerado de hiperinflación, aplicando un severo ajuste en agosto de 1990 y a desarrollar una política económica de apertura a los mercados internacionales, y a la participación del sector privado a través del fomento a la inversión de capitales extranjeros y nacionales. En esta línea se produjo la privatización de los servicios de electricidad, telefonía, entre otros, se transfirió al sector privado un gran número de empresas del Estado asumiendo este, un rol promotor y regulador.

En este marco se inicia la reforma del sector agua potable y saneamiento, con el objetivo principal de mejorar la prestación de los servicios en términos de cobertura y calidad del servicio brindado al usuario, para lo cual se establecieron las siguientes estrategias:

El rol del Estado se orienta a cumplir un rol rector, regulador y supervisor.

Se reafirma la responsabilidad de los Gobiernos Locales en la provisión de los servicios públicos.

Buscar el autofinanciamiento de las empresas de agua potable, a través de tarifas reales que cubran todos los costos operativos del servicio y costos de inversión.

Definir soluciones tecnológicas apropiadas para atender a los sectores sociales menos favorecidos, con recursos del Estado.

Fomentar la participación del sector privado.

Para implementar estas estrategias, se crea en el año 1992 la Superintendencia Nacional de Servicios de Saneamiento (en adelante, SUNASS) para cumplir el rol regulador del Estado. Bajo ella se organizan a nivel nacional las empresas como sociedades anónimas, con administración y accionariado municipal; siendo la excepción SEDAPAL quien mantuvo su status de empresa pública de propiedad del Estado.

Las acciones gubernamentales se enfocaron a la implementación de la reforma, a través de la puesta en marcha del nuevo sistema de regulación tarifaria, del sistema de fiscalización y el desarrollo de programas de inversión orientados al fortalecimiento de la gestión de las empresas de agua potable y de los organismos del sector y a la ampliación y mejoramiento de la infraestructura existente.

Posterior al año 2000, el proceso del sector se inicia con una etapa de inestabilidad política. En el campo económico se mantienen las políticas de desarrollo basadas en la búsqueda del equilibrio macroeconómico, apertura comercial internacional y mejora de la calidad del gasto y de las inversiones públicas, acciones que se han visto favorecidas por la situación internacional caracterizada por elevados términos de intercambio, crecimiento mundial y bajas tasas de interés. 
El marco legal también tuvo reformas en este período, tales como:

Simplificación del sistema tarifario desapareciendo las etapas que se venían aplicando para su implementación y se establece que la formula tarifaria se definirá de acuerdo a un Plan Maestro Optimizado (en adelante, PMO) instrumento regulatorio que permite analizar las metas, inversiones y tarifas.

Única y exclusivamente la SUNASS aprueba la fórmula tarifaria y define la tarifa, bajo criterios y principios de transparencia y audiencias públicas previas a la aprobación de aquella.

Limitar la interferencia política de los municipios, reestructurando la composición de los directorios de las empresas de agua potable permitiendo la participación de representantes de los Gobiernos Regionales y de la sociedad civil.

\section{$T$ El modelo regulatorio peruano:}

La política regulatoria en el Perú está considerando un enfoque de protección y conservación del recurso, así como la ampliación de la cobertura del servicio que impulsa el Gobierno Central. La ampliación del acceso al recurso no solo es una condición básica para la mejora de la calidad de vida, sino que va de la mano con el uso responsable del recurso, considerando que el cambio climático afecta a nivel mundial las fuentes de agua y en particular al Perú.

El regulador es consciente que, si bien el acceso al servicio significa poblaciones más saludables, el recurso debe ser protegido para que los costos de potabilización no se incrementen y se expresen en tarifas más altas para los usuarios.

Por tal motivo, la nueva visión del regulador a aplicarse con las empresas de agua potable a nivel nacional, implica la determinación de tarifas bajo un enfoque eco sistémico; es decir, promoviendo la creación de un fondo para la protección de las cabeceras de cuenca, a fin de conservar y proteger la calidad y el volumen del recurso más importante para el ser humano como es el agua.

Asimismo, el regulador viene estudiando el impacto de los nuevos mecanismos de participación público - privado en proyectos para ampliar la cobertura del servicio y mejorarla a nivel nacional, siendo prioridad la gestión sostenible del recurso. Esta situación supone un cambio normativo que adopte e incorpore nuevas figuras jurídicas, las mismas que se constituirán como el vehículo que concretice estos mecanismos y suponga el desarrollo de un sistema regulatorio célere y predecible, logrando por ende desarrollar un mercado atractivo que proporcione un alto grado de seguridad jurídica.

Todo ello representa el nuevo enfoque regulatorio que está gestionando el regulador en el sector, buscando impulsar y asentar los nuevos cimientos que permitan una mayor \más constructiva interrelación entre las empresas de agua potable y los usuarios en general.

El Perú políticamente está dividido en 24 departamentos, los cuales a su vez se subdividen en 196 provincias y 1.833 distritos. De esta cantidad de distritos, 312 se encuentran bajo el ámbito de las 50 empresas de agua potable reguladas y supervisa- 
das por la SUNASS, que tienen bajo su administración a 16,7 millones de habitantes que representan el $78 \%$ de la población urbana y $59 \%$ de la población total peruana.

\section{EL PMO COMO HERRAMIENTA DE PLANEAMIENTO Y DESARROLLO}

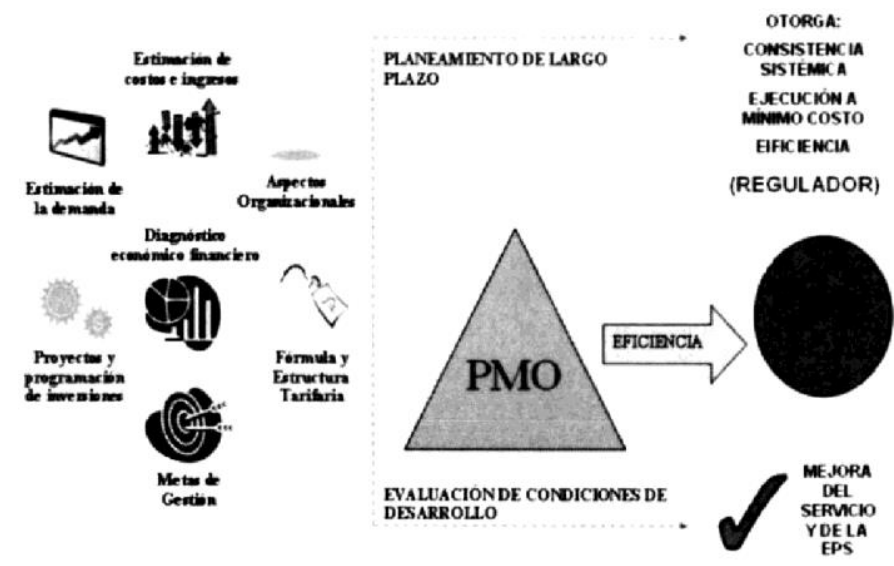

Fuente: Elaboración propia.

El marco regulatorio del sector saneamiento tiene como objetivo promover el planeamiento ordenado y oportuno de las inversiones previstas en los respectivos PMO, toda vez que de acuerdo a lo establecido en la Ley General de Servicios de Saneamiento y su Reglamento, el PMO es una herramienta de planeamiento de largo plazo que incorpora los aspectos organizacionales, el diagnóstico de la situación eco-

nómico-financiera, comercial y operacional, la estimación de la demanda, proyectos de inversión y financiamiento, la estimación de los costos e ingresos, las metas de gestión, fórmulas tarifarias, determinación de las estructuras tarifarias y la programación de las inversiones en condiciones desarrollo eficiente de las EPS. 
EI PMO analiza de manera simultánea las metas de gestión para mejorar el servicio (cobertura, calidad y eficiencia de gestión), las inversiones necesarias para alcanzar dichas metas y las tarifas que permitirán financiar las inversiones. En base al PMO, se revisa a través de niveles de micro medición, niveles de cobertura, conexiones activas y otros el cumplimiento de la empresa con las condiciones preestablecidas y de esta manera se establece la tarifa para un periodo de 5 años para que cubra los costos económicos de la empresa en la prestación de servicios y continuar con sus operaciones.

El regulador circunscribe su accionar a: (i) formular alternativas de solución para la mejora de los servicios brindados por la EPS a fin de cubrir el déficit actual y futuro de los servicios de saneamiento: (ii) brindara la EPS una señal que garantice el reconocimiento de los costos económicos, financieros y tributarios que incurra la EPS al ejecutar las soluciones de mínimo costo; $y$, (iii) asegurar la consistencia sistémica con el resto de proyectos del Programa de Inversiones contemplados en el PMO pro-

puesto por la EPS y los que han sido previstos en anteriores PMO, en el entendido que este instrumento de planeamiento tiene un horizonte de treinta (30) años ${ }^{14 .}$

\section{Los actos administrativos del regulador:}

"(...) la regulación es siempre, por definición, un sustituto del mercado; por tal motivo, las características que debe reunir deben estar orientadas a obtener los efectos benéficos y estimulantes que aquél produce, transmitir señales y mensajes que faciliten la orientación de los participantes en el sector regulado y exigir estándares de calidad y seguridad. El objetivo de la regulación no es tanto controlar a las empresas cuanto proteger a la sociedad..." ${ }^{15}$.Y es que si nos remitimos a lo desarrollado por la doctrina es necesario puntualizar

que "... las decisiones del entere regulador no revisten las caras ticas de los actos administrativos clásicos, caracterizados por lo que se llama su 'tipicidad', esto es, la vinculación estricta del contenido del acto a lo previsto por la norma en la que ese supuesto de hecho se contempla (plena subsunción del acto en la norma)..."16; por lo antes expuesto, debe entenderse que "... en la regulación económica aunque se aplique el principio de norma previa existe un margen de discrecionalidad económica y técnica. El ente regulador tiene que ponderar en muchos diferentes factores (técnicos, económicos, sociales, medioambientales, etc.) que entran en juego en toda decisión reguladora, con un impacto directo en el régimen económico de los servicios públicos..."17. En suma resulta fundamental concluir que "... el acto administrativo del ente regulador tendrá siempre

14 El regulador debe asegurar que las propuestas que formule el PMO (sin carácter mandatario) promuevan la creación de valor para la EPS y la mejora del servicio (costo-efectivo).

$15 \quad$ KRESALJA, Baldo. "Rol del estado y la gestión de los servicios públicos". En: Themis Revista de Derecho, № 39. Lima, PUCP. Página 63.

$16 \quad$ ARIÑO ORTIZ, Gaspar. "Sobre el Significado Actual de la noción de Servicio Público y su Régimen Jurídico" (Hacia un Nuevo Modelo de Regulación). En: El Nuevo Servicio Público. Madrid, 1997. Página 51.

$17 \quad$ Ibíd. 
una capacidad creadora, un ámbito de flexibilidad del que no gozan habitualmente las decisiones de la Administración ordinaria $(. .,)^{\prime 18}$.

Consecuentemente, nos encontramos en la disyuntiva si frente a una decisión del ente regulador cabe definirlo como un acto administrativo o regulatorio. Quizás el desarrollo que viene abarcando la regulación en el mundo llevaría a flexibilizar el acto administrativo, sin embargo, ello resulta típico del derecho administrativo. Las nociones económico ambientales con importantes matices cuantitativos como textos de la modernidad del derecho exigen del derecho administrativo ensayar una óptica nueva, o caso contrario, desprenderse de ella para que se genere un nuevo enfoque, aunque esto último quizás resulte difícil de asimilar en razón que son los entes estatales quienes fijan la regulación del servicio público.

Otro aspecto a considerar es lo concerniente a la asimetría de la información. Para ello debemos citar a Esther Guirlache y Richard Francés:

"(...) [s]in la existencia del problema referido a la asimetría de información, la regulación económica sería una simple cuestión de cálculos en relación a los precios óptimos, determinando las reducciones de costos que deben alcanzarse por una empresa y señalando las instrucciones para tal efecto. Esta declaración señala implícitamente el rol crucial que juega la información en el proceso regulador. Debido a sus ventajas informativas sobre las reguladoras, las empresas (...) deben revelar su potencial de eficiencia y poner en práctica la reducción de costes $(\ldots)^{\prime \prime}{ }^{19}$

Y es que la actividad regulatoria es desarrollada en medio de una ausencia considerable de información en tanto:

"(...) la regulación actúa en base a hipótesis en la mayoría de casos. Opera normalmente con información incompleta $(. . .)^{\text {m:o }}$

Así se busca reducir los efectos de dicho problema mediante las acciones de verificación y comprobación de datos que el regulador realiza y la información que la misma empresa regulada puede remitir responsablemente. En ese sentido, el acto administrativo regulatorio asume una característica fundamental adicional, que gira en tomo a la comprobación de la información cuantitativa y técnica que proporcionan los regulados sujetos a corroboración. Ello permite arrojar al final propuestas normativas administrativas y regulatorias en esencia, que tienen como protagonista a la tarifa.

18 lids

19 GERLACH, Esther y FRANCEYS, Richard. Regulating Water and Sanitation for the Poor Chaple 2: Economice Regulation.UK, 2008. Página.27. Traducción propia: "... In the absence ofinforma- lions asymmetries, economic regulalion would he a simple matler of calculaling óptima! pnces, delermin-ing cosi reduclions lo be achieved by afirm and issuing inslructions lo this effect. This statement implicity underlines the cnicial role information plav in the regulatory process. Due lo Iheir informational advan-lages over regulators, firms have to (...) reveal iheir ejficiency polenlial and implemenl cosí reduclion... "

20 BULLARD, Alfredo. Derecho y Economía. El Análisis Económico de las Instituciones Legales.

Parte VII Regulación y Libre Competencia: Ente Regulador y Agencia de Competencia: ¿Son la misma cosa? Editorial Palestra. Lima 2009. p. 907. 
— La Regulación Jurídica dE los Servicios Públicos:

Caso del agua y Saneamiento en el Perú

Para el caso del sector saneamiento en el Perú, la formulación de la tarifa encuentra sustento en los artículos $29^{\circ}$ y $31^{\circ}$ de la Ley General de Servicios de Saneamiento, los cuales establecen que:

"(...) [l]a determinación de las tarifas (...) se guía por los principios de eficiencia económica, viabilidad financiera, equidad social, simplicidad y transparencia $(\ldots)^{\prime \prime}$, y que:

"(...) [I]as fórmulas tarifarias deben reflejar los costos económicos de prestación de servicio. Estos costos consideran la eficiencia en la gestión de las entidades prestadoras, en cada uno de los sistemas

En esta misma línea, el artículo $84^{\circ}$ de su Reglamento establece que:

"(...) [l]a regulación de tarifas (...) tiene por objeto promover y asegurar la prestación de un adecuado servicio de saneamiento. Para tal fin, se establecerán tarifas que propicien la eficiencia económica $(\ldots)^{\prime \prime}$

Y, el artículo $94^{\circ}$ del mismo Reglamento al desarrollar el principio de eficiencia económica señala que:

"(...) las tarifas que cobre la EPS -empresa de servicio de agua potablepor la prestación de los servicios de saneamiento, deberán inducir a una asignación óptima de recursos que posibilite la maximización de los beneficios de la sociedad" y al referirse al principio de viabilidad financiera, el mismo artículo establece que "... las tarifas aplicadas por la EPS buscarán la recuperación de los costos requeridos para su funcionamiento eficiente, en función a los niveles de calidad y servicio que fije la Superintendencia".

Consecuentemente el regulador debe ceñirse a los principios que informan la regulación tarifaria, así como a los criterios para la definición de proyectos y las condiciones para la estructuración del financiamiento, previstos en el Reglamento General de Tarifas. Efectivamente, los proyectos que deben ser propuestos por las empresas de agua potable y. finalmente aprobados por la SUNASS, principalmente deben:

Orientarse a la solución de los problemas para la mejora de la prestación del servicio, tomando como referencia el orden de prelación siguiente: (i) mejora de la calidad del agua, (ii) mejora en el uso eficiente del recurso e infraestructura, (iii) incremento del acceso a los servicios de saneamiento, (iv) incremento de los niveles de tratamiento de aguas residuales; $y,(v)$ otros proyectos de inversión necesarios (debiéndose entender dentro de este orden de prelación a aquellos proyectos que se caracterizan por su consistencia sistémica, pues, el servicio de saneamiento es un sistema integrado y no la sumatoria de proyectos aislados). 
Determinarse en base a las conclusiones del balance oferta-demanda de cada sistema técnico a efectos de abastecer los requerimientos del servicio en el ámbito de responsabilidad de la empresa de servicio de agua potable.

Significar una solución integral de las necesidades de mejora en la prestación del servicio considerando la generación de recursos en el proyecto que permita el pago del financiamiento requerido para su implementación.

Estar formulados en base a estudios técnicos debidamente sustentados o fichas elaboradas bajo los criterios establecidos en el Sistema Nacional de Inversión Pública.

Ser tarificados si su financiamiento está asociado a la generación interna de fondos de la empresa de servicio de agua potable, créditos ya concertados debidamente acreditados por el solicitante o puedan ser financiados dentro de las restricciones de financiamiento presupuestarias y en función a la capacidad económica financiera de la empresa.

En suma, el estudio, observación, análisis y valoración de todos los componentes que la regulación tarifaria presenta no es una situación que pueda advertirse necesariamente en una fórmula normativa; no obstante, que los organismos reguladores tienen, en base a su constitución legal, la obligación de expedir disposiciones que reglamenten o normen situaciones atípicas de orden cuantitativo y cualitativo del sector en que se desenvuelven.

Ello deriva en consecuencia, en la denominada "capacidad creadora y flexible", citada por Gaspar Araño Ortiz, de la que gozan las decisiones de los organismos reguladores y que el derecho administrativo soslaya. Ameritando por ello necesariamente una amplitud en las exposiciones de motivos de sus normas aparejada bajo criterios de argumentación y transparencia que la técnica legislativa recomienda. Consecuentemente tal afirmación podría establecer la génesis de un nuevo derecho especial desprendido del derecho administrativo, producto de notas fundamentales que caracterizan a los organismos reguladores como son su especialización e independencia.

El antagonismo de este criterio, como sostiene Ramón Tupaya Tapia, es reconocer que la regulación será un fenómeno que puede ser definido desde la ciencia, la economía y la técnica, sin perder de vista que su contenido y formas, sea en la experiencia continental o anglosajona, estarán siempre vinculados al derecho, y particularmente, al derecho administrativo ${ }^{21}$.

Finalmente deberíamos considerar las disposiciones y actos administrativos que fijan la regulación tarifaria como actos administrativos clásicos o denominarlos bajo esta especialidad económica formulando una tendencia hacia un derecho regulatorio fundamentado en que la regulación económica y social y las actitudes propias del mercado generan situaciones atípicas que el derecho administrativo común no contempla; $y$, que el regulador debidamente facultado pueda generar, sobre la base de los factores antes mencionados, a través de decisiones regulatorias dotadas de la flexibilidad que carece el derecho administrativo habitual. 